\title{
Incidence and Risk Factors of Peripherally Inserted Central Catheter-related Complications
}

\author{
Cao Mingkun ${ }^{1}$, Yin Yuxia ${ }^{1,2}$, Li Xuying ${ }^{3}$, Zhang Haijun ${ }^{1,4, *}$ \\ ${ }^{1}$ National United Engineering Laboratory for Biomedical Material Modification, Dezhou, China \\ ${ }^{2}$ School of Materials Science and Engineering, University of Science and Technology Beijing, Beijing, China \\ ${ }^{3}$ Hunan Cancer Hospital, Changsha, China \\ ${ }^{4}$ Department of Vascular \& Intervention, Tenth Peoples' Hospital of Tongji University, Shanghai, China
}

Email address:

zhanghaijun@tongji.edu.cn (Zhang Haijun)

${ }^{*}$ Corresponding author

\section{To cite this article:}

Cao Mingkun, Yin Yuxia, Li Xuying, Zhang Haijun. Incidence and Risk Factors of Peripherally Inserted Central Catheter-related Complications. Biomedical Sciences. Vol. 6, No. 4, 2020, pp. 79-83. doi: 10.11648/j.bs.20200604.11

Received: September 4, 2020; Accepted: September 22, 2020; Published: October 7, 2020

\begin{abstract}
Background: Peripherally inserted central catheter (PICC) is widely used all over the world, but the complications related to it have not been well solved. Objective: To evaluate PICC-related complications and risk factors. Methods: We conducted a retrospective study at multicenters on patients who underwent PICC placement from May 2017 to June 2018 to identify the incidence and risk factors of complications. Information of patients and insertion was collected by an mobile App. Univariable and multivariable analysis were fit to examine the risk factors of complications. Odds ratios (ORs) with corresponding 95\% confidence intervals (CIs) were used to indicate the association between risk factors and complications. Results: A total of 4959 patients were included with $511(10.3 \%)$ patients developed complications. In univariable analysis, age $(P=0.026)$, smoke $(P<0.001)$, drinking $(P<0.001)$, prior surgery $(P<0.001)$ and $\mathrm{PICC}$ insertion site $(P=0.023)$ were associated with complications. Following logistic regression analysis, drinking (OR 1.72, $95 \mathrm{CI} \% 1.07-2.778, P=0.025)$ and prior surgery (OR 2.04, 95\% CI 1.55-2.67, $P<0.001$ ) remained to be independent risk factors of complications. Drinking was associated with phlebitis $(P=0.002)$ and thrombosis $(P=0.007)$, and prior surgery was associated with phlebitis $(P<0.001)$, infections $(P=0.001)$ and mechanical complications $(P=0.008)$. Conclusion: Paying more attention to patients who drink alcohol and have surgery history may reduce the risk of adverse outcome.
\end{abstract}

Keywords: PICC, Complications, Risk Factors

\section{Introduction}

PICCs are increasing used in medical practice including the infusion of total parenteral nutrition, chemotherapy and long-term intravenous fluids $[1,2]$ due to its safety and efficacy as long-term vascular access compared with central venous catheters (CVCs) [3, 4]. Despite many benefits, PICC was associated with vein thrombosis, infections, phlebitis, bleeding and mechanical complications [5-8]. Previous study reported the incidence of PICC-related complications ranging from $12 \%$ to $24.7 \%$ [9-12]. Several studies had investigated risk factors of complications. BMI [13], malnutrition, length of stay, use of anticoagulation/antiplatelet agents, after-hours placement [12] and malignancy [14] has been proved to be risk factors of complications. However, consistency has not been reached at present. So more evidence on risk factors placing patients at greater risk of complications need to be provided. Our study was performed to estimate incidence and identify risk factors of PICC-related complications.

\section{Method}

\subsection{Study Design}

In January 2019, we retrospectively studied a cohort consisted by patients who had been placed PICC from May 2017 to June 2018, regardless of the demographic, medical status or indications of patients. This multicenter retrospective cohort study was performed in 126 Chinese hospitals. After 
our screening, patients who had incomplete data (no removal date or PICC was still in use) were excluded, there were 4959 patients including in the study.

\subsection{PICC Insertion and and Post-insertion Care}

All PICCs (Cathicoid ${ }^{\mathrm{TM}}$, Branden Medical Scientific, Inc.) were $4 \mathrm{~F}$, silicone catheters. PICCs were placed using standard aseptic precaution. Portable ultrasonography was performed to evaluate a suitable insertion vein before PICC insertion. Ideal tip position of catheters was confirmed by chest X-ray. Maintainness including replacement of accessories and catheters flushing was performed weekly. All operations were executed by professional nurses team according to instructions. All patients were followed-up until they met criteria for a PICC-related complication or PICC removal.

\subsection{Variables and Definitions}

PICC-related complications include phlebitis, thrombosis, infection, bleeding, upperextremity edema and mechanical complicatios. Phlebitis was defined as an inflammation of the tunica intima of a vein [15]. Thrombosis was diagnosed by ultrasonography and professional doctors or nurses. Infection was diagnosed if presenting and growing a pathogenic microorganism [16]. Bleeding was defined as bleeding or blood exudation at the puncture point. Upperextremity edema directed any edema of unknown origin around the catheter and was diagnosed by nurses. Mechanical complications were defined according to National Cancer Institute Common Terminology Criteria for Adverse Events, version 4.0.

\subsection{Data Collection}

We collected data concerning patients' demographic characteristics, comorbidities, insertion characteristics and complication information during insertion and follow-up period by mobile App. All records on mobile App were filled out by professional nurses team.

\subsection{Statistical Analysis}

Demographic characteristics and potential risk factors were summarized using descriptive statistics: mean ( \pm standard deviation [SD]) for continuous variables and frequency (percent) for categorical variables. Chi square or Fisher exact test and logistic regression analysis were used to estimate the risk factors of PICC-related complications. Enter method was used for logistic regression models. $\alpha=0.05$ was used for the significance to enter the model, and $\alpha=0.10$ was used for significance level to delete from the model. All statistic tests were two sided and $\mathrm{P}$ value of $<0.05$ indicated statistical significance. The statistical analysis was performed with SPSS software (version 21.0, SPSS Inc., IBM, NY, USA).

\section{Results}

During May 2017 to June 2018, 4959 patients (54.2 \pm 12.8 years) at 126 multicenter was included in the study. 511 (10.3\%) patients developed PICC-related complications. Of all, $2203(44.4 \%)$ were male and $3989(80.4 \%)$ were married and there was no significant difference for gender $\left(\chi^{2}=0.00\right.$, $\mathrm{P}=0.993)$ and marriage $\left(\chi^{2}=3.57, \mathrm{P}=0.059\right)$ between patients with complications and without complications. Tract tumor $(29.3 \%)$, basilic vein $(87.0 \%)$ and supine $(99.9 \%)$ were the most frequently disease, insertion vein and puncture position, respectively. For comorbidities, patients with complications were similar to patients without complications. Univariable analysis showed that age $(\mathrm{t}=2.22, \mathrm{P}=0.026)$, smoke $\left(\chi^{2}=23.87\right.$, $\mathrm{P}<0.001)$, drinking $\left(\chi^{2}=32.41, \quad \mathrm{P}<0.001\right)$, prior surgery $\left(\chi^{2}=41.95, \mathrm{P}<0.001\right)$ and site of PICC insertion $\left(\chi^{2}=7.62\right.$, $\mathrm{P}=0.023)$ were associated with $\mathrm{PICC}$-related complications. (Table 1)

Table 1. Baseline characteristics of patients and univariate analysis of complications.

\begin{tabular}{|c|c|c|c|c|c|}
\hline & Total $N=4959$ & $\begin{array}{l}\text { Patients without complications } \\
\mathrm{n}=4448(89.7 \%)\end{array}$ & $\begin{array}{l}\text { Patients with complications } \\
\mathrm{n}=511(10.3 \%)\end{array}$ & $\chi^{2}$ & P value \\
\hline \multicolumn{6}{|l|}{ Patient characteristics } \\
\hline Age $^{*}$, year & $54.2 \pm 12.8$ & $54.4 \pm 12.7$ & $53.1 \pm 13.1$ & 2.22 & 0.026 \\
\hline Gender, Male & $2203(44.4)$ & $1976(44.4)$ & $227(44.4)$ & 0.00 & 0.993 \\
\hline Weight*, kg & $60.5 \pm 14.9$ & $60.5 \pm 15.3$ & $60.5 \pm 11.8$ & -0.01 & 0.990 \\
\hline Marriage, married & $3989(80.4)$ & $3594(80.8)$ & $395(77.3)$ & 3.57 & 0.059 \\
\hline Smoke & $417(8.4)$ & $345(7.8)$ & $72(14.1)$ & 23.87 & $<0.001$ \\
\hline Drinking & $355(7.2)$ & $287(6.5)$ & $68(13.3)$ & 32.41 & $<0.001$ \\
\hline Type of disease & & & & 3.99 & 0.408 \\
\hline Digestive tract tumor & $1453(29.3)$ & $1314(29.5)$ & $139(27.2)$ & & \\
\hline Breast cancer & $979(19.7)$ & $873(19.6)$ & $106(20.7)$ & & \\
\hline Lung cancer & $634(12.8)$ & $575(12.9)$ & $59(11.5)$ & & \\
\hline ICU status & $374(7.5)$ & $327(7.4)$ & $47(9.2)$ & & \\
\hline Other diseases & $1519(30.6)$ & $1359(30.6)$ & $160(31.3)$ & & \\
\hline \multicolumn{6}{|l|}{ Comorbidities } \\
\hline Coronary heart disease & $79(1.6)$ & $73(1.6)$ & $6(1.2)$ & 0.64 & 0.425 \\
\hline Liver cirrhosis $^{\Delta}$ & $39(0.8)$ & $37(0.8)$ & $2(0.4)$ & - & 0.427 \\
\hline Diabetes & $103(2.1)$ & $90(2.0)$ & $13(2.5)$ & 0.61 & 0.434 \\
\hline Prior surgery & $407(8.2)$ & $327(7.4)$ & $80(15.7)$ & 41.95 & $<0.001$ \\
\hline \multicolumn{6}{|l|}{ Insertion characteristics } \\
\hline Site of PICC insertion ${ }^{\Delta}$ & & & & 7.62 & 0.023 \\
\hline Right arm & $2387(48.1)$ & $2119(47.6)$ & $268(52.4)$ & & \\
\hline Left arm & $2570(51.8)$ & $2328(52.3)$ & $242(47.4)$ & & \\
\hline
\end{tabular}




\begin{tabular}{|c|c|c|c|c|c|}
\hline & Total $N=4959$ & $\begin{array}{l}\text { Patients without complications } \\
\mathrm{n}=4448(89.7 \%)\end{array}$ & $\begin{array}{l}\text { Patients with complications } \\
\mathrm{n}=511(10.3 \%)\end{array}$ & $\chi^{2}$ & P value \\
\hline Other site & $2(0.0)$ & $1(0.0)$ & $1(0.2)$ & & \\
\hline Vein of PICC insertion ${ }^{\Delta}$ & & & & 5.30 & 0.236 \\
\hline Basilic vein & $4313(87.0)$ & $3865(86.9)$ & $448(87.7)$ & & \\
\hline Brachial vein & $322(6.5)$ & $296(6.7)$ & $26(5.1)$ & & \\
\hline Median vein & $225(4.5)$ & $200(4.5)$ & $25(4.9)$ & & \\
\hline Cephalic vein & $96(1.9)$ & $85(1.9)$ & $11(2.2)$ & & \\
\hline Other veins & $3(0.1)$ & $2(0.0)$ & $1(0.0)$ & & \\
\hline Puncture position ${ }^{\Delta}$ & & & & 3.13 & 0.190 \\
\hline Supine position & 4929 (99.4) & 4423 (99.4) & $506(99.0)$ & & \\
\hline Semi decubitus position & $23(0.5)$ & $20(0.4)$ & $3(0.6)$ & & \\
\hline Sitting position & $7(0.1)$ & $5(0.1)$ & $2(0.4)$ & & \\
\hline Inserting Length*, mm & $41.1 \pm 4.2$ & $41.1 \pm 4.1$ & $40.8 \pm 5.1$ & 1.26 & 0.209 \\
\hline
\end{tabular}

${ }^{*}:$ test $^{\circ}{ }^{\Delta}$ : Fisher exact test.

The logistic regression analysis result showed drinking (OR $1.72,95 \mathrm{CI} \% 1.07-2.77, \mathrm{P}=0.025)$ and prior surgery (OR $2.04,95 \%$ CI $1.55-2.67, \mathrm{P}<0.001)$ increased the risk of PICC-related complications. Prior surgery had a greater influence on PICC-related complications. The risk of PICC-related complications for patients with surgery history was 2.04-fold compared with patients who has not undergone surgery. (Table 2)

Table 2. Logistic Regression analysis of complications.

\begin{tabular}{lllll}
\hline & $\boldsymbol{\beta}$ & OR & $\mathbf{9 5 \%}$ CI & P value \\
\hline Age & -0.01 & 0.99 & $0.99-1.00$ & 0.054 \\
Smoke & 0.18 & 1.2 & $0.75-1.90$ & 0.446 \\
Drinking & 0.54 & 1.72 & $1.07-2.77$ & 0.025 \\
Prior surgery & 0.71 & 2.04 & $1.55-2.67$ & $<0.001$ \\
Site of PICC insertion & & & & 0.086 \\
Left arm & -1.81 & 0.16 & $0.01-2.76$ & 0.209 \\
Right arm & -1.99 & 0.14 & $0.01-2.32$ & 0.169 \\
\hline
\end{tabular}

Of all 4959 patients, 138 (27.0\%), 119 (23.3\%), 89 (17.4\%), $80(15.7 \%), 51(10.0 \%)$ and 34 (6.7) patients developed phlebitis, thrombosis, infection, mechanical complications, bleeding and upperextremity edema respectively. For the relationship between risk factors and complications, drinking was related to phlebitis $(\mathrm{P}=0.002)$ and thrombosis $(\mathrm{P}=0.007)$, and surgery history was associated to phlebitis $(\mathrm{P}<0.001)$, infections $(\mathrm{P}=0.001)$ and mechanical complications $(\mathrm{P}=0.008)$. (Table 3$)$

Table 3. Relationship between complications and risk factors.

\begin{tabular}{|c|c|c|c|c|c|c|}
\hline & \multicolumn{2}{|l|}{ Drinking } & \multirow{2}{*}{$P$ value } & \multicolumn{2}{|l|}{ Prior surgery } & \multirow{2}{*}{ P value } \\
\hline & Yes, 355 (7.2) & No, 4604 (92.8) & & Yes, 407 (8.2) & No, 4552 (91.8) & \\
\hline Phlebitis, 138 (27.0) & $19(5.4)$ & $119(2.6)$ & 0.002 & $23(5.7)$ & $115(2.5)$ & $<0.001$ \\
\hline Thrombosis, 119 (23.3) & $16(4.5)$ & $103(2.2)$ & 0.007 & $13(3.2)$ & $106(2.3)$ & 0.274 \\
\hline Mechanical complications, 80 (15.7) & $10(2.8)$ & $70(1.5)$ & 0.062 & $13(3.2)$ & $67(1.5)$ & 0.008 \\
\hline Bleeding $^{\Delta}, 51(10.0)^{\prime}$ & $2(0.6)$ & $49(1.1)$ & 0.582 & $5(1.2)$ & $46(1.0)$ & 0.607 \\
\hline Upperextremity edema $^{\Delta}, 34$ (6.7) & $4(1.1)$ & $30(0.7)$ & 0.303 & $5(1.2)$ & $29(0.6)$ & 0.196 \\
\hline
\end{tabular}

${ }^{\Delta}$ : Fisher exact test.

\section{Discussion}

This multicenter retrospective cohort study aimed to estimate the incidence and risk factors of PICC-related complications. The main findings were as follows: 1) the incidence of PICC-related complication was $10.3 \%$ and phlebitis $(27.0 \%)$, thrombosis $(23.3 \%)$, infection $(17.4 \%)$ accounted for the top three complication events; 2) Drinking and prior surgery history increased the risk of PICC-related complications; 3) Drinking was related to phlebitis and thrombosis, and surgery history was associated to phlebitis, infections and mechanical complications.

A review included 19 articles reported the incidence of PICC-related complications ranged from $8 \%$ to $50 \%$ and phlebitis accounted for the largest proportion [17] which was also proved in our study. The incidence of PICC-related complications in our study was $10.3 \%$ which was lower than many reported studies [9-12, 17]. Previous studies showed catheter type [17], study object [9] and sample size [13] et al could influence the incidence of complications. The lower incidence in our study may benefit from skilled operation, better respect of the maximal sterile barrier precautions, improvement of compliance and properties of PICC. Many studies have been conducted in cancer patients $[9,18,19]$. Our study aimed to guide clinical practice regardless status of patients. Meanwhile, the sample size of this study is relatively large. Based on the above two points, this study demonstrated a reliable result that Cathicoid $^{\mathrm{TM}}$ PICC was safe for the long-term use. 
Many risk factors such as fluoropyrimidine containing chemotherapy, male gender, white cell count [20], body mass index (BMI) [13] and right side insertion have been proved in different studies. In univariable analysis, we found insertion site was risk factor of PICC-related complications which was consistent with previous study [21]. However, logistic regression analysis showed insertion site was not associated with PICC-related complications. Bashir et al have concluded insertion site had no relationship with total PICC-related complication, but was associated with mechanical complications [22]. So we can conclude insertion site may have a greater impact on one of the PICC-related complications while a lesser impact on total PICC-related complications. Logistic regression analysis indicated drinking and prior surgery were independent risk factors of PICC-related complications which had not been reported in other studies. Drinking affects vascular endothelial health. Abe et al had proved drinking may increase the risk of cardiovascular events in Dahl S rats [23]. Gardner et al [24] and Chiva et al [25] had proved ethanol chronically consumed in large amounts and chronic alcohol consuption could damage cardiovascular. A study from Croatia male indicated that a greater alcohol consumption was accompanied by higher blood pressures [26]. All these evidences suggested that drinking may lead to vascular-related complications. The mean age of patients in our study was more than 50 years old and records showed many of them had years of drinking history. Chronic alcohol consuption and greater alcohol consumption have had adverse effects on their blood vessels, further leading to vascular-related complications such as thrombosis, and phlebitis. In addition, Hansen et al [27] also found liquor consumption and binge drinking could increase the risk of venous thromboembolism. As for prior surgery, we consider that the majority of patients were malignancies which are very consumptive disease. Their health will further decline if they have undergone operations before insertion, so many complications including phlebitis, infections and mechanical complications will increase accordingly.

There are several limitations of this study. Firstly, previous studies have shown that the lumen size and durance of PICC will affect the occurrence of complications. We did not take these factors into analysis due to we did not collect relevant information by the App. More detailed information about these should be obtained. Secondly, we explored the relationship between drinking and surgery history and complications, but did not know the quantitative relationship. This deserves further study.

\section{Conclusion}

In conclusion, PICC demonstrated the safety and efficacy as long-term vascular access. But catheter-related complication could not avoided completely. More attention should be paid to patients with drinking and prior surgery history to reduce the risk of the adverse outcome. We hope that the identification of these risk factors will reduce complications and ultimately enhance patient safety and satisfaction. More researches are needed to explore factors influencing complications and clinicians need to sum up experience to prevent complications. The harm of drinking should be publicized among healthy people and the amount of drinking should be reduced as much as possible.

\section{References}

[1] HOSHAL V L, JR. Total intravenous nutrition with peripherally inserted silicone elastomer central venous catheters [J]. Archives of surgery (Chicago, Ill: 1960), 1975, 110 (5): 644-6.

[2] BOTTINO J, MCCREDIE K B, GROSCHEL D H, et al. Long-term intravenous therapy with peripherally inserted silicone elastomer central venous catheters in patients with malignant diseases [J]. Cancer, 1979, 43 (5): 1937-43.

[3] TEJEDOR S C, TONG D, STEIN J, et al. Temporary central venous catheter utilization patterns in a large tertiary care center: tracking the "idle central venous catheter" [J]. Infection control and hospital epidemiology, 2012, 33 (1): 50-7.

[4] PERIARD D, MONNEY P, WAEBER G, et al. Randomized controlled trial of peripherally inserted central catheters vs. peripheral catheters for middle duration in-hospital intravenous therapy [J]. Journal of thrombosis and haemostasis: JTH, 2008, $6(8): 1281-8$

[5] GAO Y, LIU Y, MA X, et al. The incidence and risk factors of peripherally inserted central catheter-related infection among cancer patients $[\mathrm{J}]$. Therapeutics and clinical risk management, 2015, 11 (863-71.

[6] CHOPRA V, RATZ D, KUHN L, et al. Peripherally inserted central catheter-related deep vein thrombosis: contemporary patterns and predictors [J]. Journal of thrombosis and haemostasis: JTH, 2014, 12 (6): 847-54.

[7] COADY K, ALI M, SIDLOFF D, et al. A comparison of infections and complications in central venous catheters in adults with solid tumours [J]. The journal of vascular access, 2015, 16 (1): 38-41.

[8] KANG J, SUN W, LI H, et al. Peripherally inserted central catheter-related vein thrombosis in breast cancer patients $[\mathrm{J}]$. The journal of vascular access, 2016, 17 (1): 67-71.

[9] BERTOGLIO S, FACCINI B, LALLI L, et al. Peripherally inserted central catheters (PICCs) in cancer patients under chemotherapy: A prospective study on the incidence of complications and overall failures [J]. Journal of surgical oncology, 2016, 113 (6): 708-14.

[10] COTOGNI P, BARBERO C, GARRINO C, et al. Peripherally inserted central catheters in non-hospitalized cancer patients: 5 -year results of a prospective study [J]. Supportive care in cancer: official journal of the Multinational Association of Supportive Care in Cancer, 2015, 23 (2): 403-9.

[11] PRANDONI P, POLISTENA P, BERNARDI E, et al. Upper-extremity deep vein thrombosis. Risk factors, diagnosis, and complications [J]. Archives of internal medicine, 1997, 157 (1): 57-62.

[12] MORAN J, COLBERT C Y, SONG J, et al. Screening for novel risk factors related to peripherally inserted central catheter-associated complications [J]. Journal of hospital medicine, 2014, 9 (8): 481-9. 
[13] KANG J, CHEN W, SUN W, et al. Peripherally inserted central catheter-related complications in cancer patients: a prospective study of over 50,000 catheter days [J]. The journal of vascular access, 2017, 18 (2): 153-7.

[14] CHOPRA V, ANAND S, HICKNER A, et al. Risk of venous thromboembolism associated with peripherally inserted central catheters: a systematic review and meta-analysis [J]. Lancet, 2013, 382 (9889): 311-25.

[15] PUMPO A D, BENEDETTO M, AGR F E. Infusion-Related Complications [M]. Springer Milan, 2013.

[16] SOCIETY I N. Infusion Nursing Standards [J]. J Infus Nurs, 2006, 29 (1 (Suppl)): S1-S92.

[17] SECKOLD T, WALKER S, DWYER T. A comparison of silicone and polyurethane PICC lines and postinsertion complication rates: a systematic review [J]. The journal of vascular access, 2015, 16 (3): 167-77.

[18] DUTIA M, WHITE R H, WUN T. Risk assessment models for cancer-associated venous thromboembolism [J]. Cancer, 2012, 118 (14): 3468-76.

[19] SABER W, MOUA T, WILLIAMS E C, et al. Risk factors for catheter-related thrombosis (CRT) in cancer patients: a patient-level data (IPD) meta-analysis of clinical trials and prospective studies [J]. Journal of thrombosis and haemostasis: JTH, 2011, 9 (2): 312-9.

[20] JONES D, WISMAYER K, BOZAS G, et al. The risk of venous thromboembolism associated with peripherally inserted central catheters in ambulant cancer patients $[\mathrm{J}]$. Thrombosis journal, 2017, 15 (25).
[21] PAQUET F, BOUCHER L M, VALENTI D, et al. Impact of arm selection on the incidence of PICC complications: results of a randomized controlled trial [J]. J Vasc Access, 2017, 18 (5): 408-14.

[22] BASHIR R A, SWARNAM K, VAYALTHRIKKOVIL S, et al. Association between Peripherally Inserted Central Venous Catheter Insertion Site and Complication Rates in Preterm Infants [J]. American journal of perinatology, 2016, 33 (10): 945-50.

[23] ABE C, MORITA H. Drinking-induced bradyarrhythmias and cerebral injury in Dahl salt-sensitive rats with sinoaortic denervation $[\mathrm{J}]$. Journal of applied physiology (Bethesda, Md: 1985), 2013, 115 (10): 1533-9.

[24] GARDNER J D, MOUTON A J. Alcohol effects on cardiac function [J]. Comprehensive Physiology, 2015, 5 (2): 791-802.

[25] CHIVA-BLANCH G, ARRANZ S, LAMUELA-RAVENTOS $\mathrm{R} \mathrm{M}$, et al. Effects of wine, alcohol and polyphenols on cardiovascular disease risk factors: evidences from human studies [J]. Alcohol and alcoholism (Oxford, Oxfordshire), 2013, 48 (3): 270-7.

[26] KOZAREVIC D, RACIC Z, GORDON T, et al. Drinking habits and other characteristics: the Yugoslavia Cardiovascular Disease Study [J]. American journal of epidemiology, 1982, 116 (2): 287-301.

[27] HANSEN-KRONE I J, BRAEKKAN S K, ENGA K F, et al. Alcohol consumption, types of alcoholic beverages and risk of venous thromboembolism - the Tromso Study $[\mathrm{J}]$. Thrombosis and haemostasis, 2011, 106 (2): 272-8. 